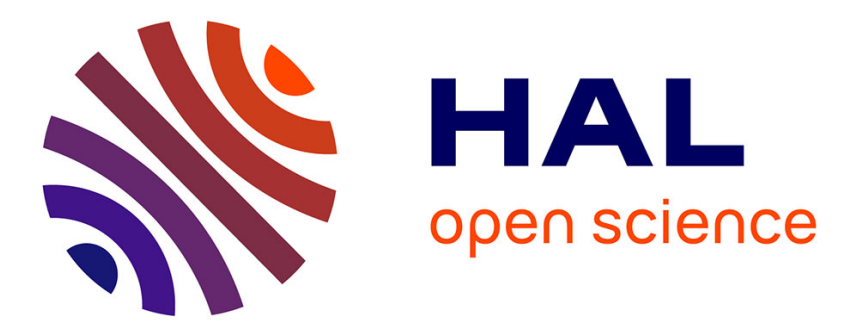

\title{
Polarized low-coherence interferometry applied to birefringent fiber characterization
}

Virginie Gaillard, Dominique Leduc, Cyril Lupi, Christian Boisrobert

\section{To cite this version:}

Virginie Gaillard, Dominique Leduc, Cyril Lupi, Christian Boisrobert. Polarized low-coherence interferometry applied to birefringent fiber characterization. Measurement Science and Technology, 2011, 22 (3), 10.1088/0957-0233/22/3/035301 . hal-01007187

\section{HAL Id: hal-01007187 https://hal.science/hal-01007187}

Submitted on 10 Mar 2018

HAL is a multi-disciplinary open access archive for the deposit and dissemination of scientific research documents, whether they are published or not. The documents may come from teaching and research institutions in France or abroad, or from public or private research centers.
L'archive ouverte pluridisciplinaire HAL, est destinée au dépôt et à la diffusion de documents scientifiques de niveau recherche, publiés ou non, émanant des établissements d'enseignement et de recherche français ou étrangers, des laboratoires publics ou privés. 


\title{
Polarized low-coherence interferometry applied to birefringent fiber characterization
}

\author{
Virginie Gaillard, Dominique Leduc, Cyril Lupi and Christian Boisrobert \\ Faculté des Sciences et des Techniques, Université de Nantes, Nantes Atlantique Universités, IREENA, \\ EA1770, 2 rue de la Houssinière-BP 9208, Nantes F-44000, France \\ E-mail: dominique.leduc@physique.univ-nantes.fr
}

\begin{abstract}
We use a polarization-maintaining low-coherence interferometer to characterize birefringent optical fibers. We show how it is possible to determine the phase and group birefringence, the orientation of the two eigenaxes and the difference of chromatic dispersions along the eigenaxes, performing three measurements only.
\end{abstract}

Keywords: interferometry, birefringence, chromatic dispersion, polarization

\section{Introduction}

Since their first industrial availability in the late 1970s, optical fibers have experienced a very important development. The emergence of microstructured fibers (also called photonic crystal fibers) is one of the very recent remarkable advances in the field of optical fibers. These fibers are made of periodic arrangements of air holes in a silica matrix [1] which give them new properties, suitable for many applications such as very high power guiding [2], supercontinuum [3, 4] or soliton links [5].

These fibers may also present some more or less detrimental characteristics in applications for which they are intended, such as birefringence [6] or chromatic dispersion $[7,8]$. Several methods have been developed to accurately measure these two characteristics, but in general not simultaneously. For the phase and group birefringence, a variety of methods have been proposed, which require measurement of spectral beat length. One can cite the method proposed by Takada et al [9], in which a modulated lateral force is applied to the fiber. This method was used to demonstrate the strong wavelength dependence of phase and group modal birefringence in photonic crystal fibers [10]. Other methods are based on an interferometric device: optical frequency domain reflectometer, to obtain the distribution of birefringence along a fiber [11], cross polarizer low- coherence interferometer [12-14] or Michelson interferometer [15].

Regarding the measurement of chromatic dispersion, there are three main methods: the time-of-flight method, the phase-shift method and interferometric methods. The latter are the most accurate for short-length samples. They were used to characterize standard fibers [16], special fibers like erbium doped fibers [17] or fiber Bragg gratings for index profile reconstruction $[18,19]$. The case of birefringent fibers is not so straightforward. Since two modes propagate along such fibers with velocities that are slightly different, the interferograms are made of two peaks, often overlapping, and beats appear in the spectrum which hinder the measurement of the chromatic dispersion. In order to make this characterization possible, one may insert a polarizer and adjust its orientation until it matches the direction of one eigenaxis of the fiber. This procedure gives good results [7, 20, 21], but is time consuming.

We propose below to use polarized low-coherence interferometry (PLCI) to characterize the birefringence, the chromatic dispersion and the orientation of the eigenaxes of a birefringent fiber. Numerical tests have been done in order to validate our method and some of these tests are presented. Finally, some results obtained on polarizationmaintaining (PM) fiber and photonic crystal fiber samples are given, showing the effectiveness of the method. 


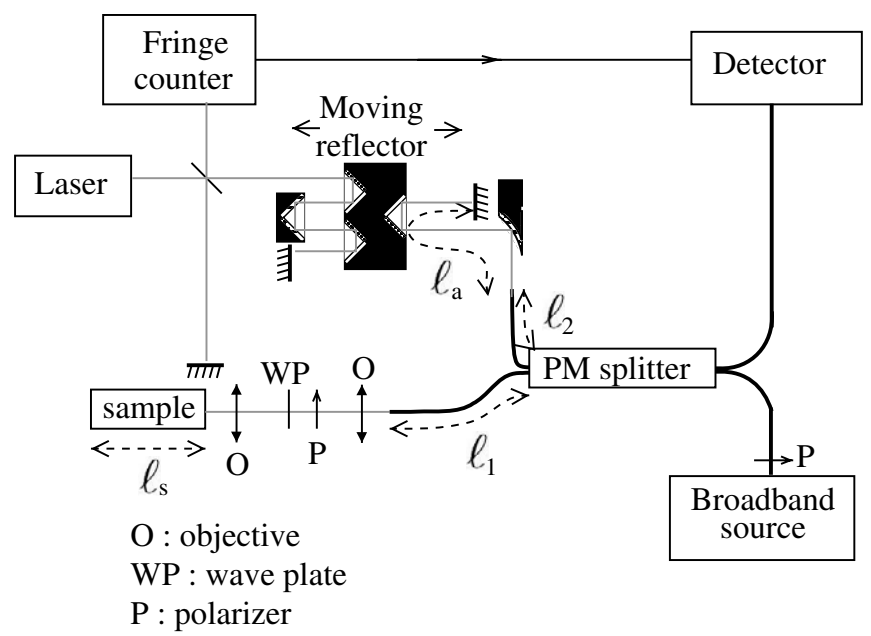

Figure 1. Polarization-maintaining low-coherence interferometer.

\section{Theory}

The low-coherence interferometer (cf figure 1) is a Michelson interferometer, realized with PM fibers [22]. All the fibers have the same orientation. Let $\vec{e}_{p}$ be the direction of one eigenaxis of the fibers. The light, emitted by an amplified spontaneous emission light source, passes through a linear polarizer, whose direction is $\vec{e}_{p}$, before being coupled to the PM fiber. It is then guided by the fiber, without change in polarization, to a PM splitter. The first output is coupled to the 'test' arm connected to the sample under test. The other output is coupled to the 'reference' arm, ending in the moving mirror. The translation of the moving reflector is monitored by a second Michelson interferometer illuminated by a highly coherent source (HeNe, frequency stabilized laser). This interferometer is used as a fringe counter and provides a regular sampling of the interferograms recorded by the broadband source interferometer.

We limit our study to linearly birefringent components: we call $\vec{e}_{x}$ and $\vec{e}_{y}$ the directions of the eigenaxes of the sample under test (figure 2) and assume that they remain constant along the sample. We then have to determine $r_{x}(\sigma) \mathrm{e}^{\mathrm{i} \phi_{x}(\sigma)}$ and $r_{y}(\sigma) \mathrm{e}^{\mathrm{i} \phi_{y}(\sigma)}$, the eigen complex reflection coefficients, and $\alpha=\left(\vec{e}_{x}, \vec{e}_{p}\right)$. There are then five unknown parameters. However, the birefringence of most fibers does not exceed $10^{-2}$ and is often lower than $10^{-3}$. This implies that the difference between the reflection coefficients $r_{x}(\sigma)$ and $r_{y}(\sigma)$, which are given by the Fresnel laws, is also lower than $10^{-2}$. This value is smaller than the experimental uncertainty due to noise. We may therefore assume that $r_{x}(\sigma)=r_{y}(\sigma)=r(\sigma)$. Moreover, for reasons that will be explained later, we will consider $\Delta \phi(\sigma)=\phi_{y}(\sigma)-\phi_{x}(\sigma)$ rather than $\phi_{x}(\sigma)$ and $\phi_{y}(\sigma)$. This reduces the five unknown parameters down to three, and then three measurements with different polarization states are required.

In order to obtain these different states, a polarizer and a wave plate (half-wave plate or quarter-wave plate) are placed at the output of the coupler, in the reference arm (cf figure 1). The orientation of the polarizer is $\vec{e}_{p}$ and the direction of one

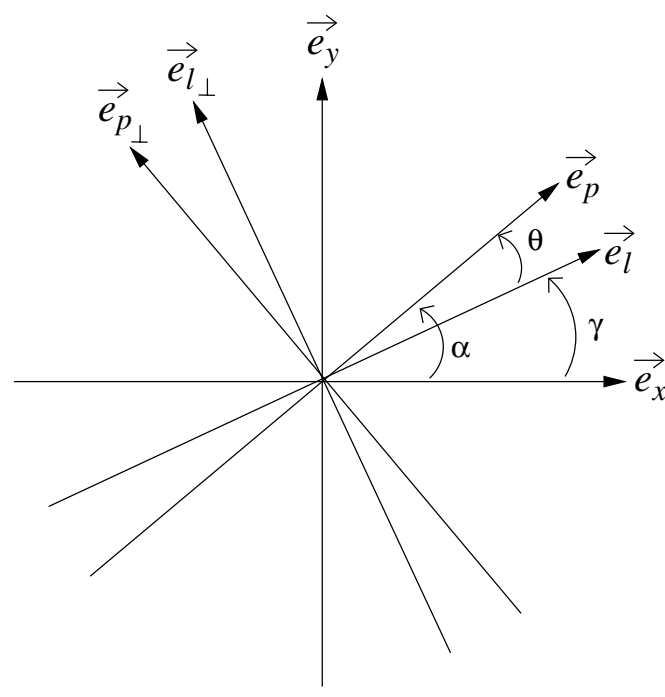

Figure 2. Diagram of the system's eigenaxis.

eigenaxis of the plate is $\vec{e}_{\ell}$. The angle between $\vec{e}_{\ell}$ and $\vec{e}_{p}$ is $\theta$. The light propagates in the air between the plate and the sample. The polarization of the light coupled to the sample is then controlled.

The electrical field at the detector level is the sum of the fields coming from both reference and test arms of the interferometer. The field from the reference arm is

$$
\vec{E}_{\mathrm{r}}(t)=\vec{e}_{p} \int_{-\infty}^{+\infty} r_{\mathrm{r}}(\sigma) \rho(\sigma) \mathrm{e}^{\mathrm{i} \phi_{\mathrm{r}}(\sigma)} \mathrm{e}^{2 \mathrm{i} \pi \mathrm{ct} \sigma} \mathrm{d} \sigma
$$

where $\sigma$ is the wavenumber, $\rho(\sigma)$ the source spectral amplitude density and $r_{\mathrm{r}}(\sigma) \exp \left[\mathrm{i} \phi_{\mathrm{r}}(\sigma)\right]$ is the reflection coefficient of the reference arm. These functions are analytic, such that $f(-\sigma)=f^{*}(\sigma)$. According to figure $1, \phi_{\mathrm{r}}(\sigma)$ can be written as

$$
\phi_{\mathrm{r}}(\sigma)=4 \pi \sigma\left[n_{\mathrm{f}}(\sigma) \ell_{2}+n_{\mathrm{a}} \ell_{\mathrm{a}}\right]
$$

with $n_{\mathrm{f}}(\sigma)=n_{0 \mathrm{f}}+\beta_{\mathrm{f}}(\sigma)$.

The field from the test arm can be derived using the Jones formalism. The propagation of the light throughout the test arm is described by the matrix $M(\sigma)$ which can be written in the standard basis $\left(\vec{e}_{p}, \vec{e}_{p \perp}\right)$ as

$$
\begin{aligned}
& M(\sigma)=M_{P} R(\theta) M_{\psi} R(\alpha-\theta) \\
& \quad \times M_{s} R(-\alpha+\theta) M_{\psi} R(-\theta) M_{P}
\end{aligned}
$$

where $M_{P}$ is the polarizer characteristic matrix, $M_{\psi}$ is the wave plate characteristic matrix

$$
M_{\psi}=\left(\begin{array}{cc}
\mathrm{i}^{\mathrm{i} \frac{\psi}{2}} & 0 \\
0 & \mathrm{e}^{-\mathrm{i} \frac{\psi}{2}}
\end{array}\right)
$$

with $\psi=\pi$ for the half-wave plate and $\psi=\pi / 2$ for the quarter-wave plate, and $M_{s}$ is the characteristic matrix of the sample under test:

$$
M_{s}=\left(\begin{array}{cc}
r(\sigma) \mathrm{e}^{\mathrm{i} 4 \pi \sigma n_{x}(\sigma) \ell_{s}} & 0 \\
0 & r(\sigma) \mathrm{e}^{\mathrm{i} 4 \pi \sigma n_{y}(\sigma) \ell_{s}}
\end{array}\right)
$$

with

$$
\left\{\begin{array}{l}
n_{x}(\sigma)=n_{0 \mathrm{~s}}+\beta_{x}(\sigma) \\
n_{y}(\sigma)=n_{0 \mathrm{~s}}+B+\beta_{y}(\sigma) .
\end{array}\right.
$$


The matrix of each component is defined in the standard basis of the component's eigenvectors. We move from one basis to another with the rotation matrices $R(\theta)$ and $R(\alpha-\theta)$. Due to the presence of the polarizers with orientation $\vec{e}_{p}$, the only component of $M(\sigma)$ different from zero is

$$
m_{11}(\sigma)=r_{\mathrm{t}}(\sigma) \mathrm{e}^{\mathrm{i} \varphi(\sigma)}\left[f_{x} \mathrm{e}^{\mathrm{i} 4 \pi \sigma n_{x}(\sigma) \ell_{\mathrm{s}}}+f_{y} \mathrm{e}^{\mathrm{i} 4 \pi \sigma n_{y}(\sigma) \ell_{\mathrm{s}}}\right]
$$

where

$$
\begin{aligned}
f_{x}= & \frac{1}{2}[1 \pm \cos 2(\alpha-\theta) \cos (2 \theta) \\
& \mp \sin 2(\alpha-\theta) \sin (2 \theta) \cos \psi]
\end{aligned}
$$

and $\varphi(\sigma)$ describes the phase due to the propagation outside the polarizing set and outside the sample. The term $r_{\mathrm{t}}(\sigma)$ is equal to $\tau(\sigma) r(\sigma)$, where $\tau(\sigma)$ is a transmission coefficient which takes into account all the losses in the test arm that do not depend on the intrinsic properties of the measured fiber (injection, angle of the cleaved end face, couplers, etc). Finally, the field from the test arm can be expressed as

$$
\vec{E}_{\mathrm{t}}(t)=\vec{e}_{p} \int_{-\infty}^{+\infty} m_{11}(\sigma) \rho(\sigma) \mathrm{e}^{2 \mathrm{i} \pi c t \sigma} \mathrm{d} \sigma .
$$

The signal recorded at the interferometer output by the photoreceiver is given by $\left\langle\left|\vec{E}_{\mathrm{r}}(t)+\vec{E}_{\mathrm{t}}(t)\right|^{2}\right\rangle_{t}$, where the brackets denote the temporal average. It is the superposition of a stationary signal corresponding to the intensities reflected by each arm and an oscillating one due to the interferences. The photoreceiver is designed to remove the stationary component; the intensity is then [19]

$$
I_{\alpha, \theta, \psi}(z)=2 \int_{-\infty}^{+\infty} \widehat{\mathcal{J}}_{\alpha, \theta, \psi}(\sigma) \mathrm{e}^{+8 \mathrm{i} \pi n_{\mathrm{a}} \sigma z} \mathrm{~d} \sigma
$$

where

$$
\widehat{\mathcal{J}}_{\alpha, \theta, \psi}(\sigma)=2 S(\sigma) \mathrm{e}^{\mathrm{i} \varphi(\sigma)}\left[f_{x} \mathrm{e}^{\mathrm{i} \phi_{x}(\sigma)}+f_{y} \mathrm{e}^{\mathrm{i} \phi_{y}(\sigma)}\right]
$$

with $S(\sigma)=r_{\mathrm{r}}(\sigma) r_{\mathrm{t}}(\sigma)|\rho(\sigma)|^{2}$ and

$$
\left\{\begin{array}{l}
\phi_{x}(\sigma)=4 \pi \sigma\left[\beta_{x}(\sigma) \ell_{\mathrm{s}}-\beta_{\mathrm{a}}(\sigma) \ell_{\mathrm{a}}+\beta_{\mathrm{f}}(\sigma)\left(\ell_{1}-\ell_{2}\right)\right] \\
\phi_{y}(\sigma)=4 \pi \sigma\left[\beta_{y}(\sigma) \ell_{\mathrm{s}}+B \ell_{\mathrm{s}}-\beta_{\mathrm{a}}(\sigma) \ell_{\mathrm{a}}+\beta_{\mathrm{f}}(\sigma)\left(\ell_{1}-\ell_{2}\right)\right]
\end{array}\right.
$$

The variable $z$ stands for the displacement of the moving reflector from the equilibrium position $\ell_{0 \mathrm{a}}$ defined as $n_{0 x} \ell_{\mathrm{s}}+$ $n_{0 \mathrm{f}} \ell_{1}=n_{0 \mathrm{a}} \ell_{0 a}+n_{0 \mathrm{f}} \ell_{2}$.

According to (10), the Fourier transform of the recorded interferogram $I_{\alpha, \theta, \psi}(z)$ is the function $\widehat{\mathcal{J}}_{\alpha, \theta, \psi}(\sigma)$. It is better to consider the squared modulus $P_{\alpha, \theta, \psi}(\sigma)$ of the functions $\widehat{\mathcal{J}}_{\alpha, \theta, \psi}(\sigma)$ which are independent of the origin of the interferograms:

$$
\begin{gathered}
P_{\alpha, \theta, \psi}(\sigma)=\left|\widehat{\mathcal{J}}_{\alpha, \theta, \psi}(\sigma)\right|^{2}=\frac{|S(\sigma)|^{2}}{2}\{[1+\cos \Delta \phi(\sigma)] \\
\left.+[1-\cos \Delta \phi(\sigma)][u(\alpha, \theta, \psi)]^{2}\right\}
\end{gathered}
$$

with $u(\alpha, \theta, \psi)=\cos 2(\alpha-\theta) \cos 2 \theta-\sin 2(\alpha-$ $\theta) \sin 2 \theta \cos \psi$.

After some algebraic manipulations, the following solutions are obtained:

$$
\cos 4 \alpha=\frac{P_{\uparrow}-P_{\nearrow}}{P_{\uparrow}+P_{\nearrow}-2 P_{\circ}}
$$

and

$$
\cos \Delta \phi(\sigma)=\frac{3 P_{\circ}-P_{\uparrow}-P_{\nearrow}}{P_{\uparrow}+P_{\nearrow}-P_{\circ}}
$$

where $P_{\uparrow}=P_{\alpha, 0, \pi}(\sigma), P_{\nearrow}=P_{\alpha, \frac{\pi}{8}, \pi}(\sigma)$ and $P_{\circ}=P_{\alpha, \frac{\pi}{4}, \frac{\pi}{2}}(\sigma)$. The functions $P_{\uparrow}$ and $P_{\nearrow}$ correspond to the linear polarizations obtained using the half-wave plate. $P_{\circ}$ corresponds to the circular polarization obtained with the quarter-wave plate.

There remains a third unknown: the reflection coefficient. The status of this parameter is slightly different, and it is not necessary to calculate it, for two reasons. First, this coefficient does not provide more information than $\Delta \phi(\sigma)$ since it also essentially depends on the refractive index of the fiber. Then, it is a very difficult parameter to measure in absolute terms. We can, of course, insert the values of $\alpha$ and $\Delta \phi(\sigma)$ obtained using equations (14) and (15) into equation (13) to determine $S(\sigma)$. But to retrieve the reflection coefficient from $S(\sigma)$, we must know the parameters $\tau(\sigma)$ and $r_{\mathrm{r}}(\sigma)$. However, there are several sources of uncertainty in the interferometer, which prevents precise knowledge of these quantities. First, each passage from free space to an optical fiber induces a strong imprecision in the amount of light injected. Then, the coupling coefficients of couplers are known with an accuracy of a few percent, corresponding to the repeatability of connections. Finally, the angle between the end face of the tested fiber and the optical axis is also a source of inaccuracy, because a small deviation from the orthogonality induces an exponential decay of the reflected intensity. For all these reasons, it is preferable to consider only relative measurements as is done in classical low-coherence interferometry.

The measurement method is based on relations (14) and (15). First of all, we may underline that the functions $P_{\uparrow}, P_{\nearrow}$ and $P_{\circ}$ depend on $\sigma$, and that $\alpha$ does not. The ratio in relation (14) is indeed independent of $\sigma$ in theory, but not in practice. Some fluctuations can be observed around an average value. We will then define $\alpha$ as

$$
\alpha=\frac{1}{4}\left\langle\arccos \left(\frac{P_{\uparrow}-P_{\nearrow}}{P_{\uparrow}+P_{\nearrow}-2 P_{\circ}}\right)\right\rangle_{\sigma}
$$

where the brackets denote the average over $\sigma$. Moreover, the relation contains the cosine function which causes an ambiguity in relation (14): if $\alpha \in\left[0 ; \frac{\pi}{4}\right]$ is a solution, then $\left(\frac{\pi}{2}-\alpha\right)$ is also a solution. This means that it is necessary to make a new measurement turning the half-wave plate of $\alpha / 2$ to determine the orientation of the eigenaxis of the sample. If the spectrum we obtained is smooth, the angle between $\vec{e}_{x}$ and $\vec{e}_{p}$ is actually $\alpha$. If the spectrum presents high beats, the real angle is $\left(\frac{\pi}{2}-\alpha\right)$.

In order to extract the birefringence $B$ and the chromatic dispersion difference $D=D_{y}-D_{x}$ from relation (15), we have to unwrap the phase which is initially folded in $[0 ; 2 \pi]$. Relying on the fact that the sample is transparent, it is possible to make a Taylor expansion of the refractive index truncating at the first order:

$$
\left\{\begin{array}{l}
n_{x}(\sigma)=n_{0 \mathrm{~s}}+a_{x}\left(\sigma-\sigma_{0}\right) \\
n_{y}(\sigma)=n_{0 \mathrm{~s}}+B+a_{y}\left(\sigma-\sigma_{0}\right)
\end{array}\right.
$$


(a)

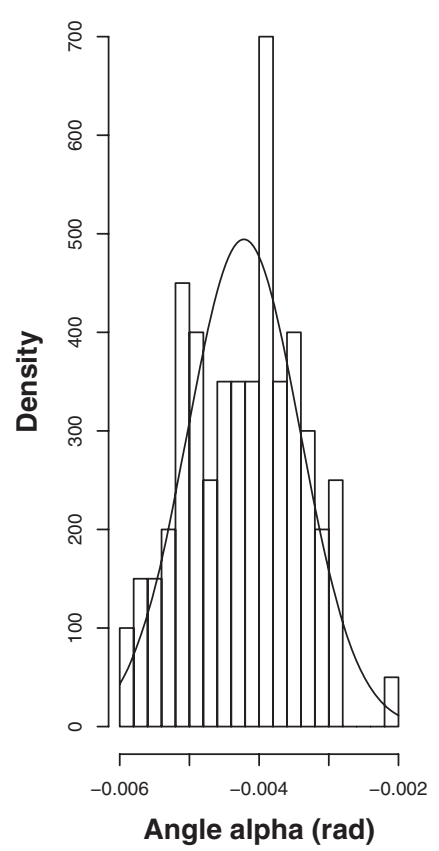

$(d)$

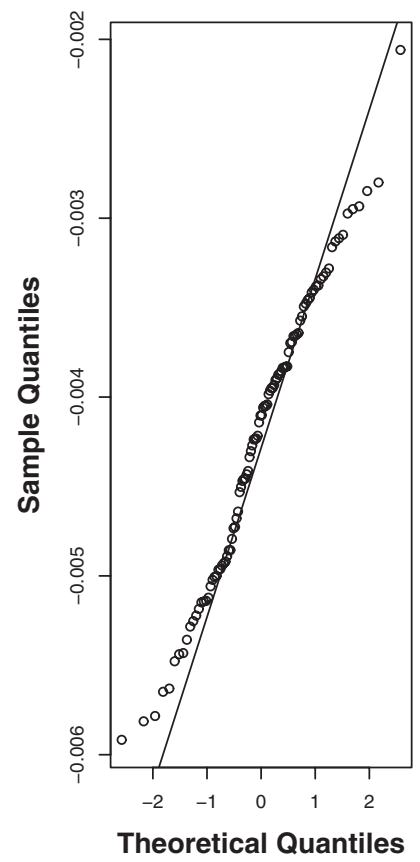

(b)

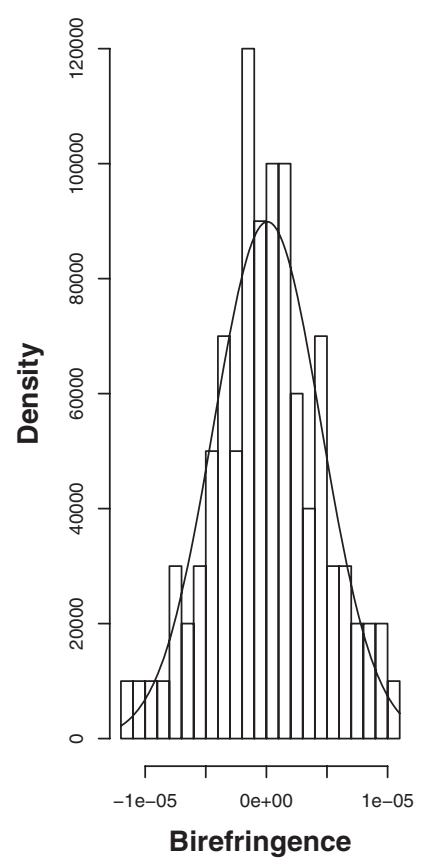

(e)

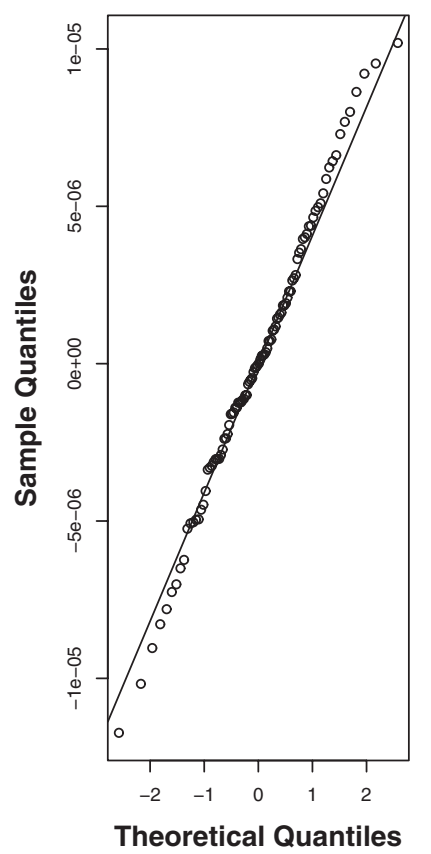

$(c)$

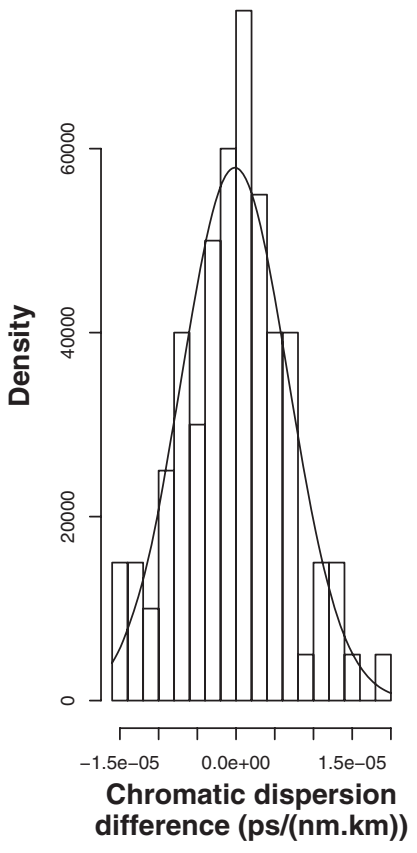

(f)

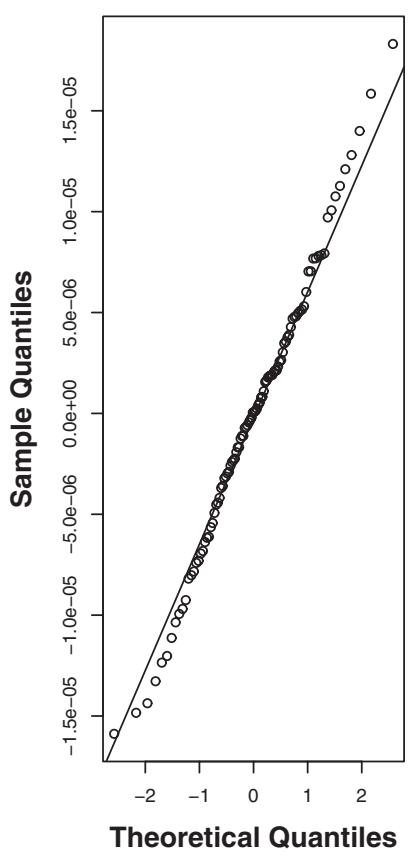

Figure 3. Distribution of the errors for $\alpha=31.3^{\circ}, B=2.2 \times 10^{-2}, D_{x}=-27.9 \mathrm{ps}(\mathrm{nm} \mathrm{km})^{-1}, D_{y}=-25.3 \mathrm{ps}(\mathrm{nm} \mathrm{km})^{-1}$ and a noise of $10 \%$.

so that

$$
\Delta \phi=4 \pi\left[B-\sigma_{0}\left(a_{y}-a_{x}\right)\right] \ell_{\mathrm{s}} \sigma+4 \pi\left(a_{y}-a_{x}\right) \ell_{\mathrm{s}} \sigma^{2} .
$$

We then need to fit the unwrapped phase with a secondorder polynomial: $a_{0}^{\mathrm{fit}}+a_{1}^{\mathrm{fit}} \sigma+a_{2}^{\mathrm{fit}} \sigma^{2}$, in order to get $a_{1}^{\mathrm{fit}}=$ $4 \pi\left[B-\sigma_{0}\left(a_{y}-a_{x}\right)\right] \ell_{\mathrm{s}}$ and $a_{2}^{\text {fit }}=4 \pi\left(a_{y}-a_{x}\right) \ell_{\mathrm{s}}$. The phase birefringence is then given by

$$
B=\frac{a_{1}^{\mathrm{fit}}+a_{2}^{\mathrm{fit}} \sigma_{0}}{4 \pi \ell_{\mathrm{s}}} .
$$

And, referring to the definition of the chromatic dispersion $D$, it can be expressed as

$$
D=\frac{a_{2}^{\mathrm{fit}}}{4 \pi c \ell_{\mathrm{s}}} \sigma^{2}
$$

Finally, the knowledge of $\alpha$ allows us to determine the chromatic dispersion of the sample along its eigenaxes. 


\section{Numerical tests}

In order to test the robustness of our method, we carried out some numerical simulations with noise.

The spectrum of the light was Gaussian, centered on $\lambda_{0}=1 / \sigma_{0}=1550 \mathrm{~nm}$ with a spectral width of $\Delta \lambda=$ $40 \mathrm{~nm}$. The angle between one eigenaxis of a sample and the direction of the polarizer was called $\alpha_{\mathrm{th}}$. The refractive indices along the eigenaxis were $n_{x \mathrm{th}}(\sigma)=n_{0 \mathrm{th}}+a_{x}\left(\sigma-\sigma_{0}\right)$ and $n_{y \mathrm{th}}(\sigma)=n_{0 \mathrm{th}}+B_{\mathrm{th}}+a_{y}\left(\sigma-\sigma_{0}\right)$. We proceeded to a random exploration of the parameter space, rather than performing a systematic exploration. To define a sample, we then chose randomly $\alpha_{\text {th }}$ in the range $\left[0 ; \frac{\pi}{4}\right], B_{\text {th }}$ in $\left[1 \times 10^{-4} ; 3 \times 10^{-2}\right]$ and $a_{x}$ in $[-0,108 \mu \mathrm{m} ; 0,108 \mu \mathrm{m}]$. The range for $\alpha_{\mathrm{th}}$ was chosen in this way because all the values in the range $\left[\frac{\pi}{4} ; \frac{\pi}{2}\right]$ would be folded in $\left[0 ; \frac{\pi}{4}\right]$. The $B_{\text {th }}$ range corresponded to what is commonly encountered. Finally, the $a_{x}$ range corresponded to a chromatic dispersion $D_{x} \in\left[-150 \mathrm{ps}(\mathrm{nm} \mathrm{km})^{-1} ; 150 \mathrm{ps}(\mathrm{nm} \mathrm{km})^{-1}\right]$, values also commonly encountered in reality. $a_{y}$ was the only parameter that was not randomly chosen. Referring to another method and related observations [21], we fixed $a_{y}=(1 \pm 0.1) a_{x}$. The sample and the source being defined, we used equation (10) to simulate the three interferograms $I_{\alpha_{\mathrm{th}}, 0, \pi}(z), I_{\alpha_{\mathrm{th}}, \frac{\pi}{8}, \pi}(z)$ and $I_{\alpha_{\mathrm{th}}, \frac{\pi}{4}, \frac{\pi}{2}}(z)$.

In our experimental protocol, we systematically proceed to ten measurements on the same sample and in the same polarization state. In order to reproduce numerically this experimental procedure, we applied different noises ten times on each theoretical interferogram, adding to each point a random quantity chosen in the range $\left[-b_{\max } ; b_{\max }\right]$. We then calculated the Fourier transforms for the ten interferograms affected by noise and determined the mean Fourier transform for each polarization. From these mean values, we calculated $P_{\uparrow}, P_{\nearrow}$ and $P_{\circ}$ and deduced $\alpha_{\text {meas }}$ and $\Delta \phi_{\text {meas. }}$. After unwrapping $\Delta \phi_{\text {meas }}$, we obtained $\mathrm{B}_{\text {meas }}$ and $D_{\text {meas }}$, and determined the errors on the different parameters:

$$
\left\{\begin{array}{l}
\delta_{\alpha}=\alpha_{\text {th }}-\alpha_{\text {meas }} \\
\delta_{B}=B_{\text {th }}-B_{\text {meas }} \\
\delta_{D}=D_{\text {th }}-D_{\text {meas }} .
\end{array}\right.
$$

We reproduced the procedure previously described a hundred times to obtain a statistical estimate of the errors. We then obtained a hundred values of $\delta_{\alpha}, \delta_{\mathrm{B}}$ and $\delta_{D}$ for one given sample. We considered the distributions of the errors and focused on the mean value denoted $\langle\delta\rangle$ and the standard deviation denoted $\sigma_{\delta}$. In figure 3 we show, as an example, the error distributions for a sample characterized by $\alpha=31.3^{\circ}$, $B=2.2 \times 10^{-2}, D_{x}=-27.9 \mathrm{ps}(\mathrm{nm} \mathrm{km})^{-1}$ and $D_{y}=$ $-25.3 \mathrm{ps}(\mathrm{nm} \mathrm{km})^{-1}$ and a noise of $10 \%$.

Statistics have been performed by the $\mathrm{R}$ software. It appears that the distributions follow a normal law, as shown by the $q-q$ plots in figures $3(d),(e)$ and $(f) . \quad \delta_{\mathrm{B}}$ and $\delta_{D}$ distributions are centered on $0\left(\left\langle\delta_{\mathrm{B}}\right\rangle \ll \sigma_{\delta_{\mathrm{B}}},\left\langle\delta_{D}\right\rangle \ll \sigma_{\delta_{D}}\right)$, $2 \sigma_{\delta_{\mathrm{B}}}$ and $2 \sigma_{\delta_{D}}$ may then be considered as the estimates of the uncertainty in $B$ and $D$. We can also observe that the mean value $\left\langle\delta_{\alpha}\right\rangle$ of the error in the angle is greater than the deviation. Therefore, we use the mean value to quantify the uncertainty in the angle.
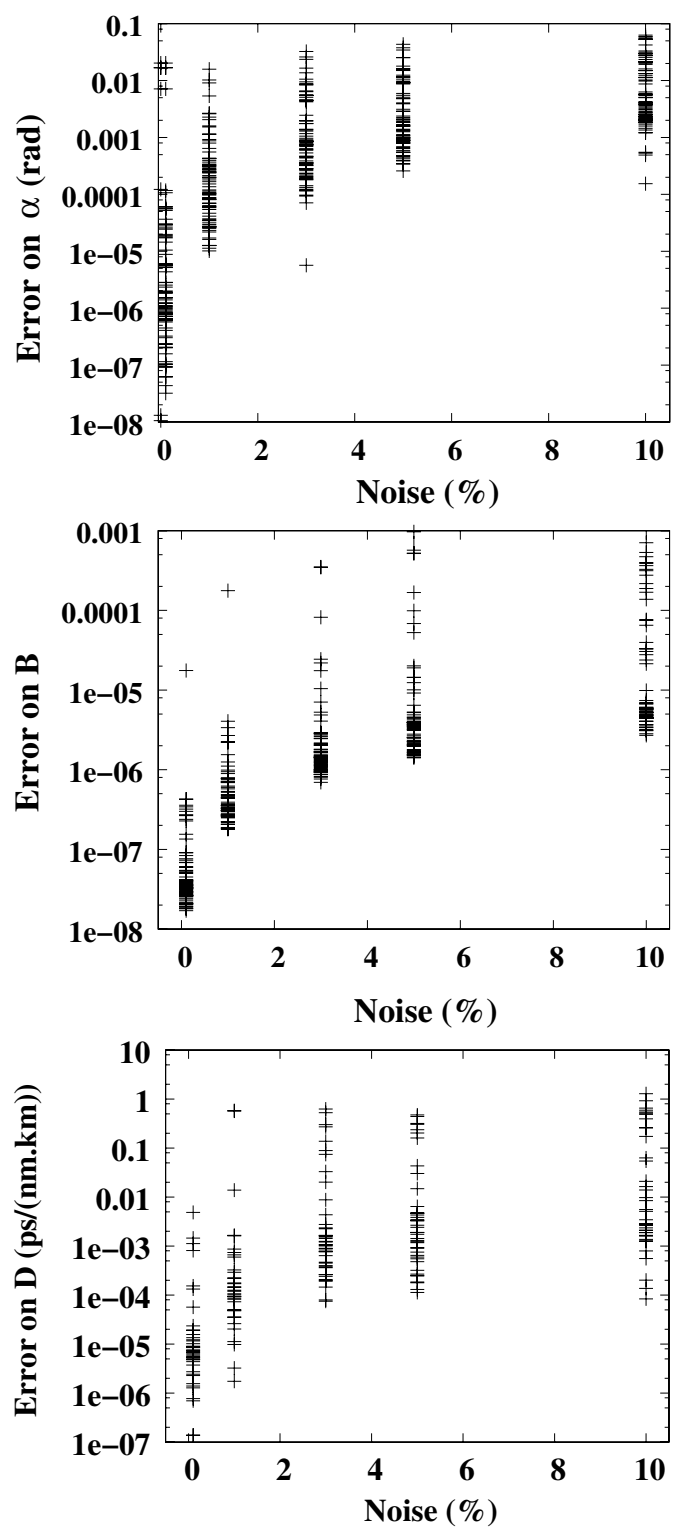

Figure 4. Evolution of the errors as a function of noise.

We performed simulations for 50 different samples. We observed no significant influence of the different parameters on the errors. The effect of noise on the different parameters is shown in the graphs of figure 4. Each cross on the graphs corresponds to a sample. As we can see, for a noise below $5 \%$ of the signal amplitude, a value commonly encountered in practice, the error in the birefringence does not exceed 0.001 ( $3 \%$ in relative value) and the error in the dispersion does not exceed 1 ps $(\mathrm{nm} \mathrm{km})^{-1}$ (5\% in relative value). The error in the angle can reach $3^{\circ}$, but this remains acceptable, knowing that the pigtailed PM components tolerance of alignment is guaranteed to be nearly $2^{\circ}$.

\section{Experimental results}

The experimental device is sketched in figure 1. Along the test arm, the light propagates in a PM fiber, is collimated by a microscope objective and propagates through a polarizer 

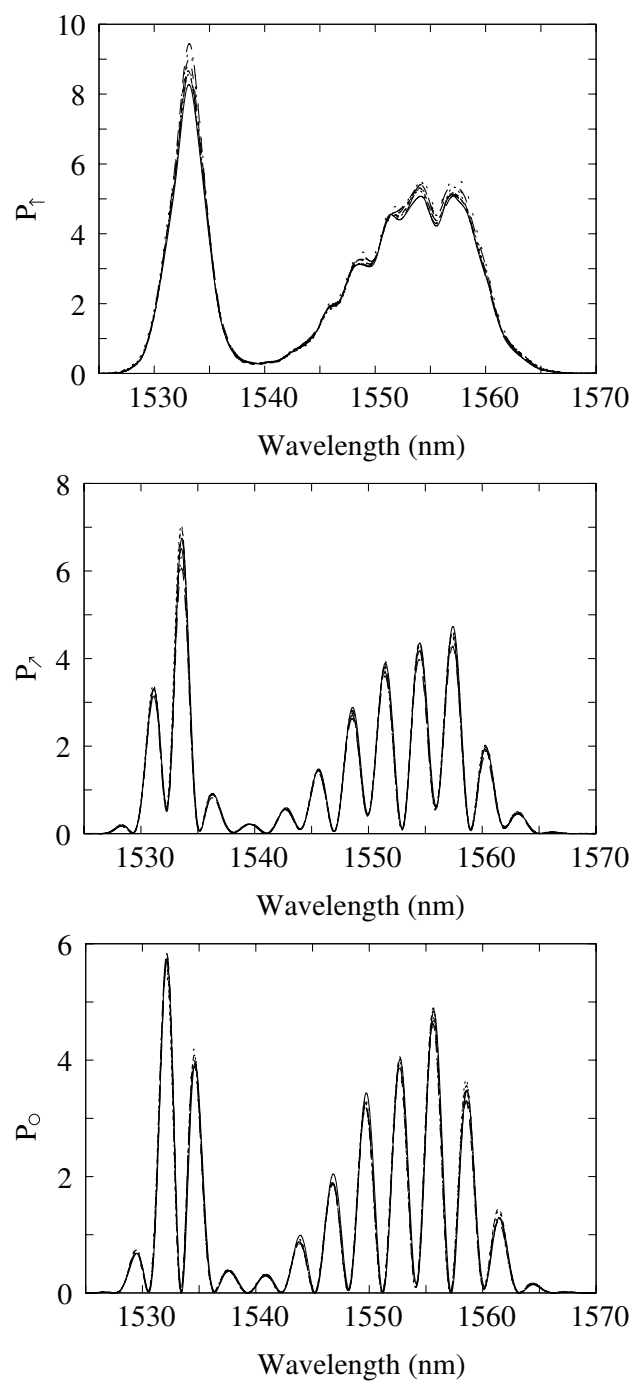

Figure 5. Functions $P_{\uparrow}, P_{\nearrow}$ and $P_{\circ}$ obtained for the Panda fiber (ten successive measurements).

and the wave plates. It is then coupled through a second objective into the fiber under test and reflected by this fiber end face. It then propagates backward along the reverse path. The propagation in free space is a delicate point of the method since it requires multiple injections into different fibers which induce important power losses and consequently decrease the signal to noise ratio.

The first measurement was performed on a standard sample: a PM fiber Panda type (Thorlabs PM1550-HP). Figure 5 shows the functions $P_{\uparrow}, P_{\nearrow}$ and $P_{\circ}$ corresponding to ten successive measurements. Characteristic beats due to the propagation of two modes appear. From these beat periods and using the classical methods [21], we determine the group birefringence $B_{g}=(4.06 \pm 0.03) \times 10^{-4}$.

From equation (16), we obtain $\alpha=20^{\circ}$. It means that $\alpha$ is actually equal to $20^{\circ}$ or $70^{\circ}$. To remove this ambiguity, we proceeded to two additional measurements: the first one using the wave plate $\lambda / 2$ oriented at $10^{\circ}$ from the polarizer axis and the second one at $35^{\circ}$ from this axis. The two spectra we observed are presented in figure 6 . The spectrum corresponding to $\alpha=70^{\circ}$ presents fewer beats, which means

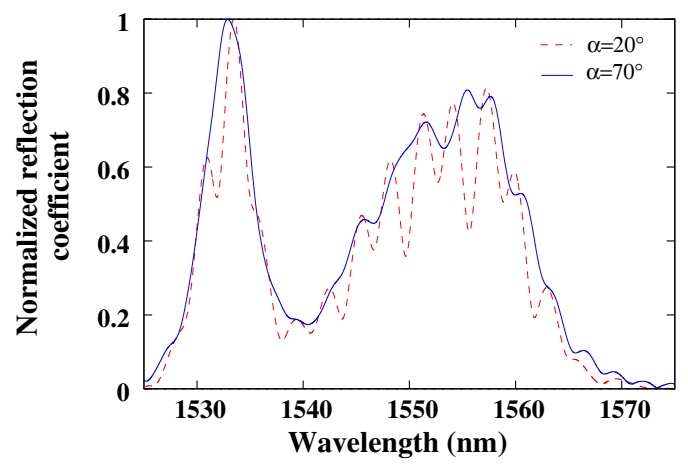

Figure 6. Fourier transform obtained for $\alpha=20^{\circ}$ and $\alpha=70^{\circ}$.

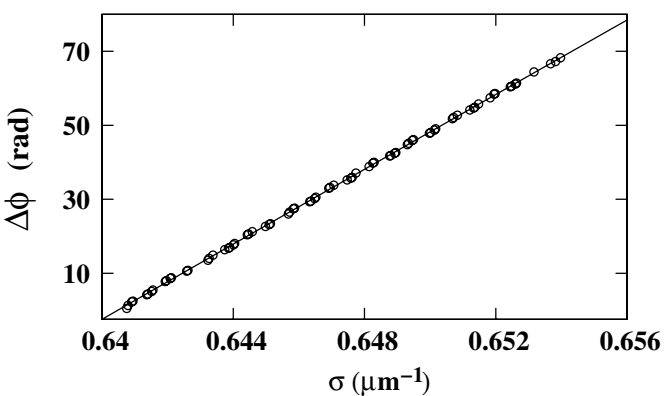

Figure 7. Unwrapped phase $\Delta \phi$ for the Panda fiber (o measurement; - fit).

this angle gives the right orientation of the eigenaxis of the fibre.

Using relation (15), we obtained the mean phase difference $\Delta \phi$ shown in figure 7. By fitting these curves with a second-order polynomial, we obtained a birefringence $B$ between $3.05 \times 10^{-4}$ and $3.17 \times 10^{-4}$ and a chromatic dispersion difference $D$ between $1.46 \mathrm{ps}(\mathrm{nm} \mathrm{km})^{-1}$ and $1.57 \mathrm{ps}(\mathrm{nm} \mathrm{km})^{-1}$. In order to have a better estimation of the uncertainty, we used the same procedure as for the numerical tests with a maximum noise of $4 \%$ : we calculated the interferogram produced by a sample with $\alpha_{\text {th }}=70^{\circ}$, $B_{\text {th }}=3.1 \times 10^{-4}$ and $D_{\text {th }}=1.43 \mathrm{ps}(\mathrm{nm} \mathrm{km})^{-1}$. This led to an uncertainty in $B$ of $0.08 \times 10^{-4}$ and in $D$ of $0.02 \mathrm{ps}$ (nm $\mathrm{km})^{-1}$. These values are comparable to the repeatability of ten measurements. We can then estimate $B=(3.11 \pm 0.08) \times$ $10^{-4}$. This value is in perfect agreement with the value given by the manufacturer which is $B=3.1 \times 10^{-4}$.

Moreover, knowing angle $\alpha$, we can measure the chromatic dispersion along the sample's eigenaxes, using a classical method [21]. Figure 8 shows the mean value of ten measured chromatic dispersions along the sample's eigenaxes called $0^{\circ}$ and $90^{\circ}$. The difference of these chromatic dispersions is plotted in figure 9 together with the chromatic dispersion difference obtained with equation (15) (solid line). Good agreement between both methods ensures the validity of the PLCI.

A second measurement has been performed on a photonic crystal fiber sample. However, the coupling in this type of fiber is not as easy as in standard fibers, which implies large losses of signal at each transition in free space. These losses induce high noise (around 20\% of the maximum of the signal 


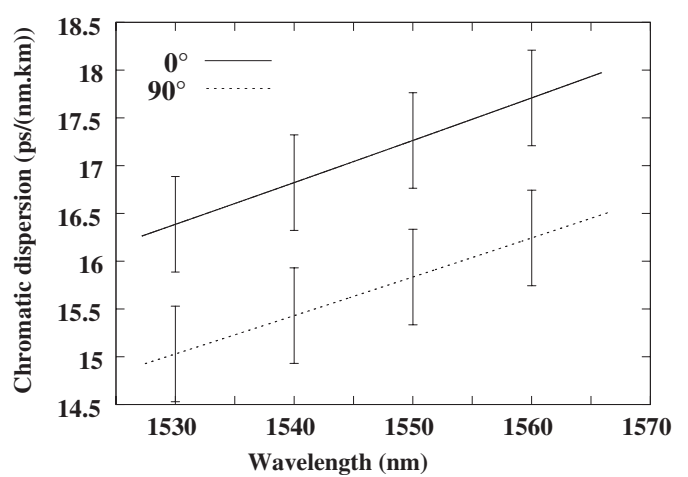

Figure 8. Mean chromatic dispersion along the Panda fiber's eigenaxes $0^{\circ}$ and $90^{\circ}$ obtained with the classical method.

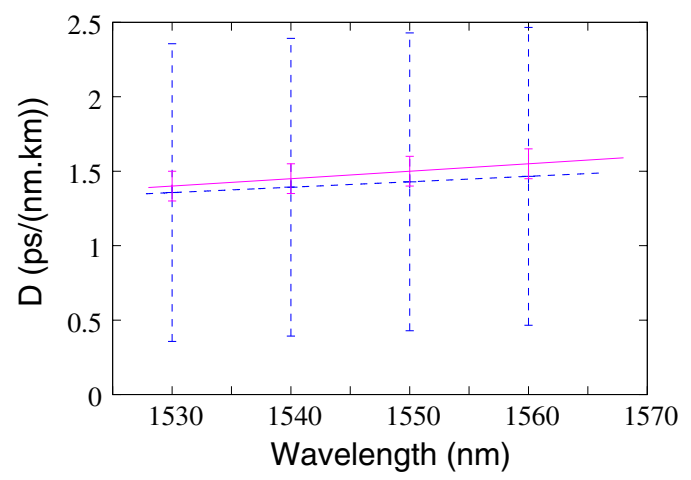

Figure 9. Chromatic dispersion difference for the Panda fiber obtained with the PLCI (solid lines) and the classical method (dashed lines).

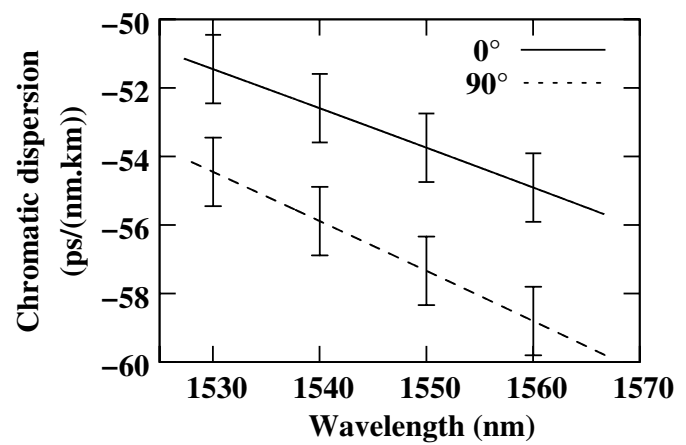

Figure 10. Mean chromatic dispersion along the microstructured fiber's eigenaxes $0^{\circ}$ and $90^{\circ}$ obtained with the classical method.

amplitude) which in turn enhances the uncertainties. The measured birefringence is $B=(8 \pm 4) \times 10^{-4}$, the angle $\alpha$ is estimated to be $24^{\circ}$. The chromatic dispersion along each eigenaxis was also measured with the classical method. Both are shown in figure 10. The differences of chromatic dispersions are plotted in figure 11. The uncertainties are estimated numerically, as before, with a noise level fixed at $20 \%$. Because of the important noise level, the slopes of the curves are somewhat different, but the difference of chromatic dispersion measured with the PLCI remains in the uncertainty range of the value measured with a classical method.

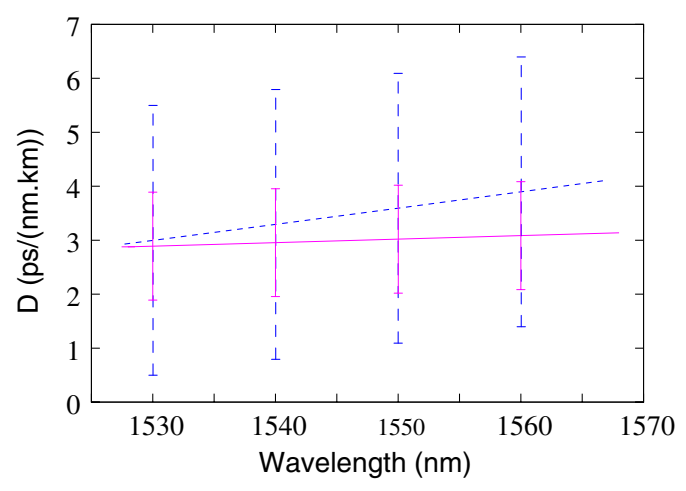

Figure 11. Chromatic dispersion difference for the microstructured fiber obtained with the PLCI (solid lines) and the classical method (dashed lines).

\section{Conclusion}

We proposed a new method for the characterization of a birefringent fiber using a polarization-maintaining lowcoherence interferometer. Basically it consists in probing the sample with three different states of light polarization and computing the square modulus of the interferogram Fourier transforms. Appropriate combinations give the orientation of the eigenaxes, the phase and group birefringence, and the difference of chromatic dispersion along the eigenaxes. Secondly, two more measurements can be performed with the light linearly polarized in the direction of each eigenaxis respectively. These measurements lead to the chromatic dispersion along the eigenaxes. The efficiency of the method was checked numerically and experimentally demonstrated afterwards on a polarization maintaining fiber and a microstructured fiber.

The accuracy of our method is similar to the accuracy of the methods described in the literature. The main advantage of our method is that the same instrument gives: the phase and group birefringence, the chromatic dispersion and the orientation of the eigenaxes of the fiber, with up to five measurements. Most of the other methods give either birefringence or chromatic dispersion, and the methods which give both require a priori knowledge of the orientation of the eigenaxes or multiple measurements to determine this orientation.

The multiple couplings from fiber to air and air to fiber, required by the wave plates in free space, are the main drawbacks of the method. However, we just have to proceed to a small number of measurements to determine the characteristics of the fiber, with a relative uncertainty of a few percent, while the noise remains smaller than $5 \%$ of the maximum of the signal's amplitude. Moreover, this method could be applied, as it is, to the characterization of planar waveguides.

\section{References}

[1] Knight J C, Birks T A, Russell P S J and Atkin D M 1996 All-silica single-mode optical fiber with photonic crystal cladding Opt. Lett. 21 1547-9 
[2] Wadsworth W J, Percival R M, Bouwmans G, Knight J C and Russell P S J 2003 High power air-clad photonic crystal fibre laser Opt. Express 1148

[3] Coen S, Hing Lun Chau A, Leonhardt R, Harvey J D, Knight J C, Wadsworth W J and Russell P S J 2001 White-light supercontinuum generation with 60-ps pump pulses in a photonic crystal fiber Opt. Lett. 26 1356-8

[4] Esposito E, Harris J, Burns D and McConnell G 2007 Measurement of white-light supercontinuum beam properties from a photonic crystal fibre using a laser scanning confocal microscope Meas. Sci. Technol. 18 2609-15

[5] Kurokawa K, Tajima K, Tsujikawa K, Nakajima K, Matsui T, Sankawa I and Haibara T 2006 Penalty-free dispersion-managed soliton transmission over a $100-\mathrm{km}$ low-loss PCF J. Lightwave Technol. 2432

[6] Bjarklev A, Broeng J, Barkou S E, Knudsen E, Søndergaard T, Berg T W and Dyndgaard M G 2000 Polarization properties of honeycomb-structured photonic bandgap fibres J. Opt. A: Pure Appl. Opt. 2 584-8

[7] Labonté L, Roy P, Pagnoux D, Louradour F, Restoin C, Mélin G and Burov E 2006 Experimental and numerical analysis of the chromatic dispersion dependence upon the actual profile of small core microstructured fibres J. Opt. A: Pure Appl. Opt. 8 933-8

[8] Ademgil $\mathrm{H}$ and Haxha S 2008 Highly birefringent photonic crystal fibers with ultralow chromatic dispersion and low confinement losses J. Lightwave Technol. 26 441-8

[9] Takada K, Noda J and Ulrich R 1985 Precision measurement of modal birefringence of highly birefringent fibers by periodic lateral force Appl. Opt. 24 4387-97

[10] Antkowiak M, Kotynski R, Nasilowski T, Lesiak P, Wojcik J, Urbanczyk W, Berghmans F and Thienpont H 2005 Phase and group modal birefringence of triple-defect photonic crystal fibres J. Opt. A: Pure Appl. Opt. 7763

[11] Froggatt M E, Gifford D K, Kreger S, Wolfe M and Soller B J 2006 Characterization of polarization-maintaining fiber using high-sensitivity optical-frequency-domain reflectometry J. Lightwave Technol. 24 4149-54

[12] Ortigosa-Blanch A, Knight J C, Wadsworth W J, Arriaga J, Mangan B J, Birks T A and Russell P St J 2000 Highly birefringent photonic crystal fibers Opt. Lett. 25 1325-7
[13] Michie A, Canning J, Lyytikainen K, Åslund M and Digweed J 2004 Temperature independent highly birefringent photonic crystal fibre Opt. Express 125160

[14] Labonté L, Pagnoux D, Roy P, Bahloul F and Zghal M 2006 Numerical and experimental analysis of the birefringence of large air fraction slightly unsymmetrical holey fibres $O p t$. Commun. 262 180-7

[15] Hlubina P, Martynkien T and Urbańczyk W 2003 Dispersion of group and phase modal birefringence in elliptical-core fiber measured by white-light spectral interferometry $O p t$. Express 11 2793-8

[16] Rao Y-J and Jackson D A 1996 Review Article. Recent progress in fibre optic low-coherence interferometry Meas. Sci. Technol. 7 981-99

[17] Gaillard V, Aduriz X, Daher N, Chapeleau X, Leduc D, Lupi C, Traynor N, Casari P and Boisrobert C 2009 Local and spectral characterization of optical fibers and fiber Bragg gratings with low coherence interferometry Fiber Integr. Opt. 28 108-26

[18] Giaccari P, Limberger H and Salathé R 2003 Local coupling coefficient characterization of fiber Bragg gratings $O p t$. Lett. 28 598-600

[19] Leduc D, Chapeleau X, Lupi C, Gejo F L, Douay M, Le Ny R and Boisrobert C 2007 Experimental synthesis of fiber Bragg gratings index profiles: comparison of two inverse scattering algorithms Meas. Sci. Technol. 18 12-8

[20] Palavicini C, Jaouën Y, Debarge G, Kerrinckx E, Quiquempois Y, Douay M, Lepers C, Obaton A and Melin G 2005 Phase-sensitive optical low-coherence reflectometry technique applied to the characterization of photonic crystal fiber properties Opt. Lett. 30 361-3

[21] Gaillard V, LaLouat L, Lupi C, Leduc D, Provino L, Traynor N, Nguyen T N, Chartier T, De Fornel F and Boisrobert C 2007 Optical characterization of photonic crystal fibers by optical low coherence reflectometry and scanning near field optical microscopy Proc. SPIE $\mathbf{6 5 8 8}$ $65880 \mathrm{~L}$

[22] Noda J, Okamoto K and Sasaki Y 1986 Polarizationmaintaining fibers and their applications J. Lightwave Technol. 4 1071-89 